\title{
Food Traceability and Authenticity Based on Volatile Compound Analysis
}

\author{
Dufossé Laurent
}

\section{Introduction}

Between December 2014 and January 2015, Operation Opson IV, an Interpol and Europol coordinated operation, seized more than 2,500 tons of counterfeit, contaminated and adulterated food, in raids across 47 countries. The seizures included mozzarella cheese, strawberries, eggs, cooking oil and dried fruits. Previously Opson III, in the summer of 2014, similarly resulted in the seizure of 1,200 tons of fake or substandard foods and nearly 430,000 liters of counterfeit drinks (oil, vinegar, biscuits, spices and condiments, cereals, dairy products and honey) (Masters 2015).

Lest anyone be in any doubt, the adulteration, falsification, counterfeiting and mis-selling/labelling of food and drinks, of ingredients, is a huge problem for the food and drink industry. Finding technical, and practical solutions to thwart the wrongdoers, is also a major challenge. It should be clear that there is no single test or "black-box" able to fix the analysis or verification of an authentic food or drink (Masters 2015). As explained in this book, many techniques are in use or are emerging and this chapter focuses on volatile compound analysis, through gas chromatography, mass spectrometry, and even (bio)electronic noses.

\footnotetext{
Laboratoire de Chimie des Substances Naturelles et des Sciences des Aliments, ESIROI Agroalimentaire, Université de La Réunion, F-97490 Sainte-Clotilde, Ile de La Réunion, France; E-mail: laurent.dufosse@univ-reunion.fr
} 


\section{Some Words about Volatile Compounds, Gas Chromatography, Mass Spectrometry and other Techniques}

The whole set of foods produced in the world contains thousands of volatile compounds. As an example the chemical identities and CAS/FEMA-GRAS / EC numbers (if known) of more than 7,100 flavor chemicals, the qualitative and the quantitative occurrence of these flavor chemicals in about 450 food products, linked to more than 4,500 references (including reviews) related to these products are described in databases such as Volatile Compounds in Food 16.2 from Triskelion ${ }^{\mathrm{TM}}$.

Studying food traceability and authenticity is not only a question of analytical chemistry as data handling using statistical tools and soft wares is at least as important as chemistry to obtain useful conclusions. Up to now the best analytical combination is based on gas chromatography (GC) and mass spectrometry (MS) applied on the headspace (HS) of the food product, avoiding the tedious and artifact-introducing extraction step. Developments in the solid phase micro extraction (SPME) also brought huge improvements in the laboratories.

Such techniques are however very costly (huge initial investment and maintenance) and perhaps users will see in the next decade applications of bioelectronic noses that are a mix of nanotechnologies and biological components, as previous e-noses did not perform very well, with their quite primitive sensors.

\section{Food Traceability and Authenticity Based On Volatile Compound Analysis: Applications in Various Foods and Drinks}

\subsection{Honey}

Assessment of the botanical origin of honey is of great concern in food analysis, since authenticity (i) guarantees the quality of the product, (ii) prevents overpayment and (iii) helps to identify frauds. In addition, European Community legislation concerning honey, i.e., Directive EC/74/409 amended by the Proposal COM/95/0722 (1997) requires labelling of the floral origin. Usually the determination of the botanical origin of honey is carried out by melissopalynological analysis, which is based on the identification of pollen by microscopic examination (Bianchi et al. 2005). This analysis is time-consuming, requires a very skillful analyst for data interpretation and sometimes results do not allow a reliable identification of the botanical origin, especially in the case of honey characterized by low amounts of pollen. Consequently, due to difficulties in analysis, great attention has been paid in order to find specific chemical markers for proving the botanical source of honeys. One of the most typical features 
of honey is its aroma profile, which could be used to characterize volatile marker compounds specific to a given botanical origin. In particular, the gas chromatography pattern can be considered as a chemical "fingerprint", since the nature and relative amount of the compounds present in the volatile fraction are a distinctive feature of different floral sources (Bianchi et al. 2005). Volatile compounds in honey may arise, among others, from the nectar or honeydew source, from the transformations of these raw materials carried out by honey bees, from honey processing and storage or from microbial and environmental contamination.

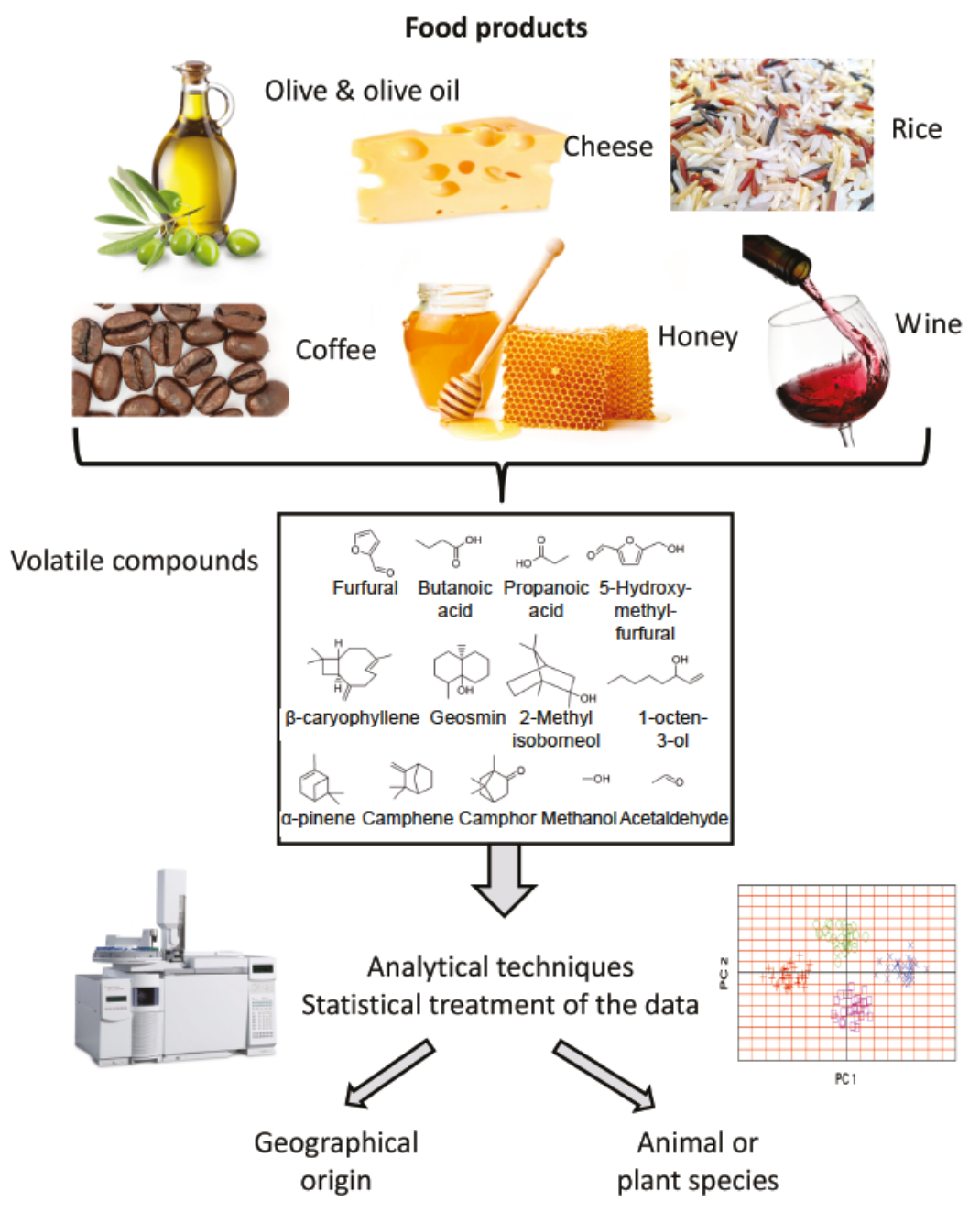

Figure 1. Description of the process, allowing determination of food traceability and authenticity through volatile compound analysis. 
Gas chromatography coupled to mass spectrometry (GC-MS) combines the high sensitivity and efficacy required for the analysis of the very complex mixtures of volatiles present in honey at low concentrations and provides structural information (mass spectrum) for their qualitative analysis. Although GC-MS has become the technique of choice for characterization of the volatile fraction of honey, its application requires a previous fractionation step in which volatiles are isolated from the major components of the honey matrix (sugars and water) and pre-concentrated (Soria et al. 2008). Several studies have been published on the application of different fractionation techniques to the study of honey volatiles: solvent extraction (SE), simultaneous steam distillation-extraction (SDE), mixed proceduresbased on SE followed by SDE, solid phase extraction, static headspace, solid phase dynamic extraction (SPDE), solid phase micro-extraction (SPME) and dynamic headspace (Purge and Trap system, P\&T).

Up to over 100 to 110 volatiles with different functionality have been identified in honey samples. Norisoprenoid compounds such as $\alpha$-isophorone, $\beta$-isophorone and 4 -oxoisophorone, were recognized as specific floral origin markers of the strawberry-tree (Arbutus unedo L.) honey. In heather (Calluna vulgaris) the presence of isophorone and 2-methyl butyric acid and the absence of linalool were quite characteristic (Seisonen et al. 2015). The occurrence of lilac aldehyde [2-(5-methyl-5-vinyltetrahydro-2furanyl) propanal] and 2-aminoacetophenone were proposed as indicators of rhododendron honey (Senyuva et al. 2009). Excess amount of volatiles such as thymol (trace) and carvacrol $(0.66 \%)$ that originate from thyme plants indicated adulteration of thyme honey by thyme essential oil in some commercial samples (Mannaş and Altuğ 2007). In another study on the volatiles of coriander, lime and sunflower honeys, 3-hexen-1-ol, isoeugenol, cinnamaldehyde, cinnamyl alcohol, pyridine, quinoline, salicylic aldehyde, undecanol and octanol were identified as the major compounds. The characteristic aroma components of buckwheat honey were shown to be 3-methylbutanal, sotolon and $\beta$-damascenone, whereas thistle, tulip, sourwood and mountain laurel honeys were found to involve volatiles such as benzaldehyde, furfural, isovaleraldehyde and phenylacetaldehyde (Mannaş and Altuğ 2007).

As a conclusion often given by the authors in this research field, multivariate statistical analysis has to be applied to GC-MS relative data, with promising results being obtained for the characterization of honey source.

\subsection{Coffee}

Coffee is the world most popular beverage after water, considering that over 400 billion cups are consumed annually. Consequentially, coffee is one of the most important agricultural products in the international trade. 
More than 800 volatile compounds have been identified to be present in roasted and ground coffee. The most common classes of compounds reported in the headspace include acids, aldehydes, alcohols, sulphur compounds, phenolic compounds, pyrazines, pyridines, thiophenes, pyrroles, ketones, esters, benzenic compounds, thiazoles, oxazoles, lactones, alkanes, alkenes, and furans (Yang et al. 2016). These components are present in variable concentrations and each of them contributes uniquely to the final aroma quality (see the excellent Master of Science in Chemistry thesis from Risticevic 2008).

Research dedicated to the recognition of coffee adulteration practices has been conducted for many years. Regarding the adulterate addition of roasted grains to coffee, the following complete list of ways to perform fraudulent practices as pertaining to coffee is: (i) adulteration with coffee substitutes (such as roasted soybean and wheat added in ground roasted); (ii) mixing two coffee bean varieties and (iii) mixing of high commercial value coffee beans from one region with lower commercial value ones originating from other regions (Risticevic 2008).

HS-SPME-GC-TOFMS methodology was developed and optimized for the purposes of verifying its capability in terms of tracing back the coffee samples to their production areas (Zambonin et al. 2005). Among the tested commercially available fiber coatings, the mixed-phase DVB/ CAR/PDMS fiber demonstrated best performance characteristics for a wide range of analytes having different physico-chemical characteristics and hence this coating was used in super elastic metal fiber assembly form for the completion of overall sequence of coffee samples. The SPME method optimization was completed by the utilization of multivariate experimental design and accordingly the optimum set of conditions for the two identified influential parameters was $55^{\circ} \mathrm{C}$ for $12 \mathrm{~min}$ for extraction time and temperature, respectively (Risticevic et al. 2008). The utilization of high-speed data acquisition rate option offered by the TOFMS instrument ensured the completion of one GC-MS run of a complex coffee sample in $7.9 \mathrm{~min}$. The complete list of benefits provided by TOF software, including the fully automated background subtraction, baseline correction, peak finds and mass spectral deconvolution algorithms were exploited during the data evaluation procedure. Finally, the acquired data set was submitted to principal component analysis and the corresponding geographical origin discriminations of coffees originating from South and Central America, Africa and Asia were successfully established (Risticevic et al. 2008).

At this point it is important to emphasize that in addition to successful geographical discrimination of: (i) authentic sample collections from Brazil and Colombia and (ii) non-authentic sample collections from South America, Central America, Africa and Asia, this classification study was also successful in detecting potential compositional changes that coffee 
undergoes due to the limited shelf-life stability over extensive storage conditions. This finding is crucial in the realization that imported food commodities are quite likely to undergo authentic aroma losses before they are distributed to consumer populations. Finally, the conducted geographical origin verification of collected samples proved that this rapid analytical methodology demonstrates great potential for the assessment of quality and detection of adulterations in worldwide coffee industry (Risticevic et al. 2008).

\subsection{Wine}

Wine aroma is formed by a complex mixture of many natural and processing variables, such as varietal origin, degree of maturity at harvest, percent of solid present in the fermented grape juice, wooded/unwooded process and bacterial/yeast strains (Cozzolino et al. 2005). A great number of papers have been published about the classification of wines according to geographical origin, based on their volatile compounds.

As an example the work of Berna et al. (2009) deals with Sauvignon Blanc wines. Analysis of 34 Sauvignon Blanc wine samples from three different countries and six regions were performed by gas chromatographymass spectrometry (GC-MS). Linear discriminant analysis (LDA) showed that there were three distinct clusters or classes of wines with different aroma profiles. Wines from the Loire Region in France and Australian wines from Tasmania and Western Australia were found to have similar aroma patterns. New Zealand wines from the Marlborough region as well as the Australian ones from Victoria were grouped together based on the volatile composition. Wines from South Australia region formed one discrete class (Berna et al. 2009). There are similarities qualitatively and quantitatively in the volatile profile of wines from different regions. Seven analytes, most of them esters (ethyl ethanoate, nerol oxide, 1-hexanol, trans-2-hexenyl butanoate, pentyl ethanoate, hex-4-enoic acid ethyl ester and propyl decanoate), were found to be the relevant chemical compounds that characterized the classes (Berna et al. 2009). However, this technique was not strong enough to sort all the six wines. Isotope mass spectrometry (IRMS) seems to be the most suitable complementary technique.

\subsection{Spirits}

The production of fermentation ethanol and spirits is an important branch of the agrofood industry in the world. The quality and the price of these products are determined by the variety, purity and origin of the raw material (Bauer-Christoph et al. 1997). National food and customs regulations have to be respected when checking the quality of these products for marketing 
and sales and also for official control. There have always been attempts to adulterate spirits and other food products, for instance by blending high-quality distillates with ethanol made from a cheaper raw material, by adding synthetic, volatile components to neutral alcohol or by misleading labelling of the variety and origin of the raw material used (Bauer-Christoph et al. 1997).

Determination of the botanical origin of raw spirit used for alcoholic beverage production is of great importance for rectifying units, control laboratories, and proper product labeling. Raw spirit samples produced from rye, corn, and potato were analyzed using a solid phase microextractionmass spectrometry (SPME-MS) method, which involved volatiles preconcentration by SPME with subsequent volatile fraction characterization by MS without particular compounds separation by GC (Jeleń et al. 2010). The main groups of volatile fermentation by-products that raw spirits contain are carbonyl compounds, higher alcohols (fusel alcohols or oils), esters, and acetals. Some of them, such as fusel alcohols and ethyl acetate, are present in relatively large amounts (often several hundred milligrams per liter) and can be determined by direct injection of the sample without a pre-concentration step, whereas others, such as carbonyl compounds (except acetaldehyde) and fatty acid ethyl esters, occur at microgram per liter concentrations and require specific isolation or detection procedures. Obtained data were treated using principal component analysis and linear discriminant analysis (LDA) to test the possibility of sample classification. SPME sampling conditions allowed rapid extraction in $2 \mathrm{~min}$ at $50^{\circ} \mathrm{C}$ using a carboxen/divinylbenzene/polydimethylsiloxane fiber, followed by rapid MS analysis. Use of LDA made possible the classification of raw spirits based on the material they were produced from. The classification ability of the developed SPME-MS method was $100 \%$, whereas its predictive ability was $96 \%$ (Jeleń et al. 2010).

\subsection{Vinegar}

Sherry vinegar and traditional balsamic vinegar (TBV) are high-quality vinegars that are famous all over the world. Sherry wine vinegar is produced in a series of wooden oak casks in which acetification and aging take place simultaneously. TBV is obtained by a traditional method that ferments the cooked must and ages the product in a set of wooden barrels (Cerezo et al. 2009). During this period, the finished product develops the desired organoleptic properties that make it highly appreciated.

The EU-funded WINEGAR project (ref. COOP-CT-2005-017269) tested four different kinds of woods (acacia, chestnut, cherry, and oak) for wine vinegar production. The quality and chemical composition were checked throughout the process and can be used for a variety of purposes, including 
authentication and product classification according to quality criteria. For example, the analysis of the vinegars aged in acacia (Robinia pseudoacacia) revealed a compound that was not present in vinegars aged in the other woods. This compound named (+)-dihydrorobinetin was likely to serve as a chemical marker of acacia wood for authenticity purposes (Cerezo et al. 2009).

\subsection{Rice}

Basmati rice is taken here as an example. There is increasing concern regarding the authenticity of basmati rice throughout the world. For years, traders have been passing off a lesser quality rice as the world's finest longgrained, aromatic rice, Basmati, in key markets like the US, Canada, and the EU. A DNA rice authenticity verification service in India has concluded that more than $30 \%$ of the basmati rice sold in the retail markets of the US and Canada is adulterated with inferior quality grains. In Britain, the Food Standard Agency found in 2005 that about half of all basmati rice sold was adulterated with other strains of long-grain rice (Cleland et al. 2014). Waters ${ }^{\mathrm{TM}}$ Company developed a complete set of methods to assess the authenticity of basmati rice using off the shelf supermarket samples with the latest advancements in high resolution GC-MS hardware and informatics. Based on 2907 statistically significant ions from the 3885 originally generated, the authors conclude they are able to discriminate basmati rice, jasmine rice, long grain rice and any combination of these (Cleland et al. 2014).

\subsection{Olive oil}

Olive oil represents an important ingredient in the popular Mediterranean diet and is worldwide appreciated both for nutritional and sensory properties, often related to geographical origin and cultivar of olive fruits employed. Fraudsters trying to seek financial gain can adulterate the product causing economic repercussions and, sometimes sanitary risks. The "protected designation of origin" (PDO) European label insures a relative protection of both consumers and honest producers, since it prescribes production techniques and specific geographical origin, but one of the main problems is to set down objective tools to control these specifications (Janin et al. 2014). The sensorial attributes of olive oils are mainly generated by volatile constituents, including carbonyl compounds (aldehydes, ketones, etc.), alcohols, esters and hydrocarbons. These attributes may have a high influence on the flavor of olive oil. Thereby, the potential discrimination of geographical origin of olive oil can be based on their volatile profile characterization (Cerrato Oliveros et al. 2005). In some cases, up to 65 
volatile compounds (aldehydes, alcohols, furans, hydrocarbons, acids, ketones, esters) have been listed and compared to show that virgin olive oil authentication is closely related with the olive variety from which oil is obtained by strictly physical means (Aparicio et al. 1997). One variety could show the maximum concentration of esters, responsible for the green (grass) perception, while another one has the maximum concentration of furans, which are responsible for sweet (ripe fruit) perception. In the frame of oil characterization, the techniques analyzing volatiles have been applied with success for the determination of adulteration such as mixing of olive oil with hazelnut oil, sunflower oil and/or olive-pomace oil, for the detection of contaminants (such as benzene, toluene, ethylbenzene, xylene isomers and styrene) (Janin et al. 2014).

\subsection{Cheese}

The origins of volatile compounds found in cheese are diverse and can be classified into two groups: the first one contains native volatile compounds already present in milk, which are not transformed during cheese manufacturing while the second group includes components produced in the cheese itself during manufacture or maturation. Forage is an important factor influencing the composition of the volatiles of the first group. The volatile compounds of the second group are formed during manufacture and ripening of cheese by microbial, enzymatic and (bio) chemical transformations (Pillonel et al. 2003). As a first example, the volatile compounds of Emmental cheese samples from different European regions were investigated by GC-MS. Each region could be separated from the others using compounds which were more or less specific to one or two regions. For instance, the concentrations of butan-2-one, 3-hydroxybutanone, butan-2-ol and octane made it possible to separate "Switzerland" origin from the other cheeses. These investigations showed the potential of volatile compounds to discriminate cheese samples with different origins (Pillonel et al. 2003).

Analysis of the volatile fraction of Provola dei Nebrodi, a typical Sicilian pasta filata cheese, was performed using Solid Phase Micro-extraction HighResolution Gas Chromatography/Mass Spectrometry. The cheeses were sampled and analyzed at four different stages of ripening $(0,7,30$ and 90 days). A total of 60 components were identified: fatty acids (11), esters (15), lactones (2), methyl ketones (8), aldehydes (9), alcohols (4), hydrocarbons (3), terpenes (1), chlorinated compounds (1), and aromatic compounds (6). The main components were found to be hexanoic and octanoic acids and ethyl hexanoate. The 60 components were present in all the samples, regardless of the ripening stage, although their ratio showed statistically significant variations with aging. Fatty acids and esters increased, whereas the aldehyde content drastically decreased after 30 days. The profile of 
volatile components identified appeared to be different from that of other pasta filata cheeses (Ziino et al. 2005).

In another case, the limited number of volatile compounds measured in the headspace of the Montasio cheese, a typical protected designation of origin (PDO) Italian semi-hard and semi-cooked cheese produced in northeast Italy from raw or thermized cow's milk, did not allow clear determination of origin. However the information could be useful in preserving this cheese in the market and provides an additional means to evaluate the effects of new technologies or changes in the traditional production techniques described in the PDO regulations for this cheese (Innocent et al. 2013).

\subsection{Meat}

Besides sensory tests of meat flavor, the instrumental determination of volatile compounds and their profile is an alternative. In order to determine the geographic origin of meat, especially of processed meat, studying the volatile compounds occurring in the headspace and in meat itself could be interesting. Gas chromatography (GC) combined with mass spectroscopy (MS) and olfactometry was used to determine and identify volatile compounds in Italian-type dry-cured meat products, but could not describe single-flavor compounds eliciting the typical salami or Parma ham flavor. There have also been several studies using different methods investigating volatile compounds in raw and cooked chicken meat and during spoilage (Senter et al. 2000), or monitoring the stage of ripening from volatile components in salami (Franke et al. 2005). Particularly promising is the analysis of volatile compounds for the determination of the geographic origin in the case of processed meat. It has to be emphasized that, in this case, these compounds code for the site where the processing is done and not for the origin of the raw meat, as these sites are not necessarily identical. Processing would add flavors, e.g., from bacteria, smoke or air, which do not only characterize the specific product, but also may be specific for the geographic origin. This approach, therefore, seems unsuitable for unprocessed meat where the concentration of volatile compounds is very low (Franke et al. 2005).

\section{Electronic Nose (E-Nose), Bioelectronic Nose}

Since the first applications of solid state gas sensors in arrays, some thirty three years ago, "electronic noses" (e-noses) have undergone a great deal of development. Around 4800 articles on this subject have been published on all aspects and among them about 850 specifically for food testing. 


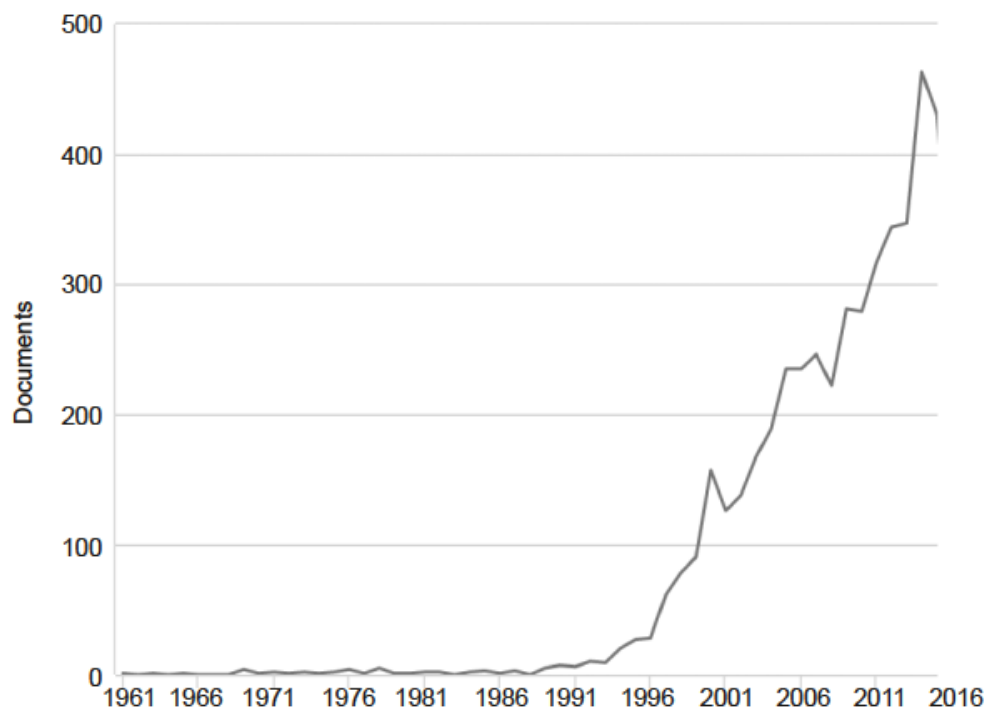

'Electronic' AND 'nose' in Scopus database Total 4838 documents

with 'food' term added

842 documents

database prospecting

done on Sept 8, 2016

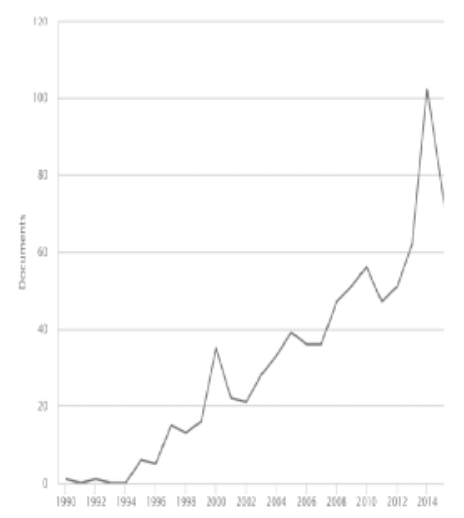

Figure 2. Bibliometrics about e-noses (Scopus database query done on September 8, 2016).

Future will tell us whether bioelectronic nose will surpass sensor array technology.

The name "electronic nose" comes from a certain parallel of the measurement concept of the instrument and that of the mammalian olfactory system (Ampuero and Bosset 2003), even if some authors reject such a name, and prefer sensor array technology. Electronic noses base the analysis of the cross-reactivity of an array of semi-selective sensors. Hence, products with similar aroma generally result in similar sensor response patterns (similar "fingerprints") whereas products with different aroma show differences in their patterns (different "fingerprints"). The interaction 
of volatiles with the array of sensors provokes a series of signals which are then processed by the computer via a pattern recognition program. Just like the human olfactory system, electronic noses do not need to be specially designed to detect a particular volatile. In fact, they can learn new patterns and associate them with new odors via training and data storage functions as humans do (Ampuero and Bosset 2003).

However, the electronic nose has many limitations to overcome (low sensitivity, low selectivity) and appear now to be something from the past. Recently, the bioelectronic nose (Lim and Park 2014), using biological components, has been developed. The bioelectronic nose seems to have a bright prospect as a powerful and effective biosensing system, capable of detecting and discriminating a huge variety of odorant molecules (Lim et al. 2013). The most meaningful characteristics of the bioelectronic nose are that it mimics the human olfactory system. The bioelectronic nose is expected to replace the sensory evaluation method. It can be used for standardization of smell, development of code for each smell, and visualization of smell (Ko et al. 2014). Consequently, the development of the bioelectronic nose is expected to open up many new possibilities to improve the procedures for assessment of authenticity and traceability of food, when problems such as cost and limited service life will be solved.

\section{Conclusion and Future Perspectives}

The past two decades have seen the advent of high throughput technologies in biology, making it possible to sequence genomes cheaply and quickly, to measure gene expression for thousands of genes in parallel, and to test large numbers of potential regulatory interactions between genes in a single experiment. The large amounts of data created by these technologies have given rise to entire new research areas in biology, such as computational biology and systems biology. The latter, which attempts to understand biological processes at a 'systems' level, is particularly indicative of the potential advantage that large datasets and their analysis can offer to biology (Ahnert 2013), and to other fields of research such as food chemistry. Thanks to big data in the cloud, making big data technology more accessible regardless of expertise or budget, we can expect to see more application of data analytics throughout the food industry in the future (Thusoo 2014). Based on thousands of food flavor tests done every day, millions and billions food flavor investigations dataset, it will increase our capacity to determine food traceability and authenticity based on volatile compound analysis. 


\section{Abbreviations}

CAS : CAS Registry Number, also referred to as CASRN or CAS Number, is a unique numerical identifier assigned by the Chemical Abstracts Service (CAS) to every chemical substance described in the open scientific literature.

EC : European Community, European Community number for chemicals within EU regulatory schemes.

FEMA : Flavor and Extract Manufacturers Association. Expert Panel of FEMA is the primary body for the safety evaluation of food flavoring for the flavor industry and the public through its assessment of flavoring substances.

GRAS : "GRAS" is an acronym for the phrase Generally Recognized As Safe. Under sections 201(s) and 409 of the USA Federal Food, Drug, and Cosmetic Act (the Act), any substance that is intentionally added to food is a food additive, that is subject to premarket review and approval by FDA, unless the substance is generally recognized, among qualified experts, as having been adequately shown to be safe under the conditions of its intended use, or unless the use of the substance is otherwise excepted from the definition of a food additive.

HS : Headspace. The air or empty space left above the contents in a sealed container, where flavor compounds could be extracted.

LDA : Linear discriminant analysis (LDA) is a generalization of Fisher's linear discriminant, a method used in statistics to find a linear combination of features that characterizes or separates two or more classes of objects or events.

MS : Mass spectrometry. An instrumental method for identifying the chemical constitution of a substance by means of the separation of gaseous ions according to their differing mass and charge-also called mass spectroscopy.

PCA : Principal Component Analysis. Principal component analysis (PCA) is a statistical procedure that uses an orthogonal transformation to convert a set of observations of possible correlated variables into a set of values of linear uncorrelated variables called principal components.

PDO : Protected Designation of Origin. Among the three European Union schemes of geographical indications and traditional specialties, known as protected designation of origin (PDO), protected geographical indication (PGI), and traditional specialties guaranteed (TSG) that promote and protect names of quality agricultural products and foodstuffs. 
SPME : Solid Phase Micro Extraction. Solid-phase micro extraction, or SPME, is a solid phase extraction sampling technique that involves the use of a fiber coated with an extracting phase, that can be a liquid (polymer) or a solid (sorbent), which extracts different kinds of analytes (including both volatile and non-volatile) from different kinds of media, that can be in liquid or gas phase. The quantity of analyte extracted by the fiber is proportional to its concentration in the sample as long as equilibrium is reached or, in case of short time pre-equilibrium, with the help of convection or agitation.

TOF : Time-Of-Flight. Time-of-flight mass spectrometry (TOFMS) is a method of mass spectrometry in which an ion's mass-tocharge ratio is determined via a time measurement. Ions are accelerated by an electric field of known strength. http:// www.kore.co.uk/tutorial.htm

\section{References}

Ahnert, S.E. 2013. Network analysis and data mining in food science: the emergence of computational gastronomy. Flavour 2: 4.

Ampuero, S. and Bosset, J.O. 2003. The electronic nose applied to dairy products: a review. Sensors and Actuators B: Chemical 94(1): 1-12.

Aparicio, R., Morales, M.T. and Alonso, V. 1997. Authentication of European virgin olive oils by their chemical compounds, sensory attributes, and consumers' attitudes. Journal of Agricultural and Food Chemistry 45(4): 1076-1083.

Bauer-Christoph, C., Wachter, H., Christoph, N., Roßmann, A. and Adam, L. 1997. Assignment of raw material and authentication of spirits by gas chromatography, hydrogen- and carbon-isotope ratio measurements I. Analytical methods and results of a study of commercial products. Zeitschriftfür Lebensmittel-Untersuchung und -Forschung A 204(6): 445-452.

Berna, A.Z., Trowell, S., Clifford, D., Cynkar, W. and Cozzolino, D. 2009. The geographical origin of Sauvignon Blanc wines predicted by mass spectrometry and metal oxide based electronic nose. Analytica Chimica Acta 648(2): 146-152.

Bianchi, F., Careri, M. and Musci, M. 2005. Volatile norisoprenoids as markers of the botanical origin of Sardinian strawberry-tree (Arbutus unedo L.) honey: Characterisation of aroma compounds by dynamic headspace extraction and gas chromatography-mass spectrometry. Food Chemistry 89: 527-532.

Cerezo, A.B., Espartero, J.L., Winterhalter, P., Garcia-Parrilla, M.C. and Troncoso, A.M. 2009. (+)-Dihydrorobinetin: a marker of vinegar aging in acacia (Robinia pseudoacacia) wood. Journal of Agricultural and Food Chemistry 57(20): 9551-9554.

Cerrato Oliveros, C., Boggia, R., Casale, M., Armanino, C. and Forina, M. 2005. Optimization of a new headspace mass spectrometry instrument. Discrimination of different geographical origin olive oils. Journal of Chromatography A 1076(1-2): 7-15.

Cleland, G., Ladak, A., Lai, S. and Burgess, J. 2014. The use of HRMS and statistical analysis in the investigation of Basmati rice authenticity and potential food fraud. Waters ${ }^{\mathrm{TM}}$ application note 10 pages. 
Cozzolino, D., Smyth, H.E., Cynkar, W., Dambergs, R.G. and Gishen, M. 2005. Usefulness of Chemometrics and mass spectrometry-based electronic nose to classify Australian white wines by their varietal origin. Talanta 68(2): 382-387.

European community. 1974. Council Directive 74/409/EEC on 22 July 1974 on the harmonization of the laws of the Member States relating to honey.

European community. 1997. Report on the Commission proposal concerning the simplification of the vertical directives on food. Proposals for Council directives relating to: (i) certain sugars intended for human consumption (COM(95)0722 - C4-0402/96 - 96/0113 (CNS), (ii) honey (COM(95)0722 - C4-0403/96 - 96/0114 (CNS)), (iii) fruit juices and certain similar products intended for human consumption (COM(95)0722 - C4-0404/96-96/0115 (CNS)), (iv) certain partly or wholly dehydrated preserved milk for human consumption (COM(95)0722 - C4-0405/96 - 96/0116(CNS)), (v) fruit jams, jellies and marmalades and chestnut purée intended for human consumption (COM(95)0722 - C4-0406/96 96/0118(CNS)). Committee on the Environment, Public Health and Consumer Protection.

Franke, B.M., Gremaud, G., Hadorn, R. and Kreuzer, M. 2005. Geographic origin of meatelements of an analytical approach to its authentication. European Food Research and Technology 221(3-4): 493-503.

Innocente, N., Munari, M. and Biasutti, M. 2013. Characterization by solid-phase microextraction-gas chromatography of the volatile profile of protected designation of origin Montasio cheese during ripening. Journal of Dairy Science 96(1): 26-32.

Janin, M., Medini, S. and Técher, I. 2014. Methods for PDO olive oils traceability: state of art and discussion about the possible contribution of strontium isotopic tool. European Food Research and Technology 239(5): 745-754.

Jeleń, H.H.,Ziółkowska, A. and Kaczmarek, A. 2010. Identification of the botanical origin of raw spirits produced from rye, potato, and corn based on volatile compounds analysis using a SPME-MS method. Journal of Agricultural and Food Chemistry 58(24): 12585-12591.

Ko, H.J., Lim, J.H., Oh, E.H. and Park, T.H. 2014. Applications and perspectives of bioelectronic nose. pp. 263-283. In: Bioelectronic Nose: Integration of Biotechnology and Nanotechnology. ISBN 9789401786133.

Lim, J.H., Park, J., Ahn, J.H., Jin, H.J., Hong, S. and Park, T.H. 2013. A peptide receptor-based bioelectronic nose for the real-time determination of seafood quality. Biosensors and Bioelectronics 39: 244-249.

Lim, J.H. and Park, T.H. 2014. Concept of bioelectronic nose. pp. 1-22. In: Bioelectronic Nose: Integration of Biotechnology and Nanotechnology. ISBN 9789401786133.

Mannaş, D. and Altuğ, T. 2007. SPME/GC/MS and sensory flavor profile analysis for estimation of authenticity of thyme honey. International Journal of Food Science and Technology 42: $133-138$.

Masters, K. 2015. How technology can weed out the fraudsters. The World of Food Ingredients 4: $55-56$.

Pillonel, L., Ampuero, S., Tabacchi, R. and Bosset, J.O. 2003. Analytical methods for the determination of the geographic origin of Emmental cheese: Volatile compounds by GC/ MS-FID and electronic nose. European Food Research and Technology 216(2): 179-183.

Risticevic, S. 2008. HS-SPME-GC-TOFMS methodology for verification of geographical origin and authenticity attributes of coffee samples. Master of Science in Chemistry thesis, 101 pages, Waterloo, Ontario, Canada.

Risticevic, S., Carasek, E. and Pawliszyn, J. 2008. Headspace solid-phase microextraction-gas chromatographic-time-of-flight mass spectrometric methodology for geographical origin verification of coffee. Analytica Chimica Acta 617: 72-84.

Seisonen, S., Kivima, E. and Vene, K. 2015. Characterization of the aroma profiles of different honeys and corresponding flowers using solid-phase microextraction and gas chromatography-mass spectrometry/olfactometry. Food Chemistry 169: 34-40. 
Senter, S.D., Arnold, J.W. and Chew, V. 2000. APC values and volatile compounds formed in commercially processed, raw chicken parts during storage at 4 and $13^{\circ} \mathrm{C}$ and under simulated temperature abuse conditions. Journal of the Science of Food and Agriculture 80(10): 1559-1564.

Senyuva, H.Z., Gilbert, J., Silici, S., Charlton, A., Dal, C., Gürel, N. and Cimen, D. 2009. Profiling Turkish honeys to determine authenticity using physical and chemical characteristics. Journal of Agricultural and Food Chemistry 57(9): 3911-3919.

Soria, A.C., Martínez-Castro, I. and Sanz, J. 2008. Some aspects of dynamic headspace analysis of volatile components in honey. Food Research International 41: 838-848.

Thusoo, A. 2014. How big data is revolutionizing the food industry. Wired https://www. wired.com/insights/2014/02/big-data-revolutionizing-food-industry/.

Yang, N., Liu, C., Liu, X., Kreuzfeldt Degn, T., Munchow, M. and Fisk, I. 2016. Determination of volatile marker compounds of common coffee roast defects. Food Chemistry 211: 206-214.

Zambonin, C.G., Balest, L., De Benedetto, G.E. and Palmisano, F. 2005. Solid-phase microextraction-gas chromatography mass spectrometry and multivariate analysis for the characterization of roasted coffees. Talanta 66: 261-265.

Ziino, M., Condurso, C., Romeo, V., Giuffrida, D. and Verzera, A. 2005. Characterization of "ProvoladeiNebrodi", a typical Sicilian cheese, by volatiles analysis using SPME-GC/ MS. International Dairy Journal 15(6-9): 585-593. 